\title{
Revista de la
}

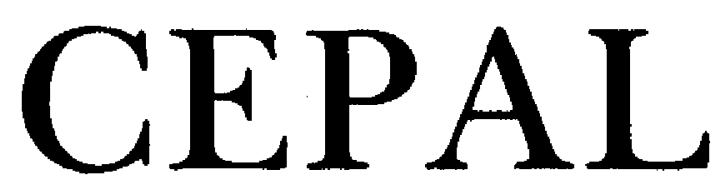

Secretario Ejecutivo

Gert Rosenthal

Secretario Ejecutizo Adjunto

Carlos Massad

\section{Director de la Revista}

Aníbal Pinto

SecretariotTécnico

Eugenio Lahera

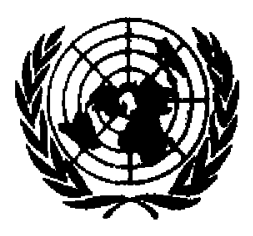

NACIONES UNIDAS

COMISION ECONOMICA PARA AMERICA LATINA Y EL CARIBE SANTIAGO DE CHILE, AGOSTO DE 1991 


\section{Revista de la}

\section{CEPAL}

Santiago de Chile

Agosto de 1991

Número 44

\section{SUMARIO}

Reconversión industrial, apertura comercial y papel del Estado en Centroamérica.

Larry Wilmore y Jorge Mattar.

Perspectivas sobre la equidad. Marshall Wolfe.

Actores sociales y ajuste estructural. Eugenio Tironi y Ricardo A. Lagos.

La selectividad como eje de las políticas sociales. Percy Rodríguez Noboa

El regreso de América Latina al mercado crediticio privado internacional. Peter West.

Conversión de la deuda externa en capital. Michael Mortimore.

Opciones para la reactivación latinoamericana en los años noventa. Colin Bradford.

Relación entre productividad media y productividad agrícola. Gerardo Fujü.

Los nuevos escenarios internacionales. Emesto Ottone.

Contaminación industrial y urbana: opciones de política. Hernán Durán.

Inserción internacional e innovación institucional. Fernando Fajnzylber.

Orientaciones para los colaboradores de la Revista de la CEPAL.

Publicaciones recientes de la CEPAL. 
REVISTA DE LA CEPAL N $\mathbf{N}^{\mathfrak{4} 4}$

\section{Opciones para la reactivación latinoamericana en los años noventa}

\author{
Colin I. Bradford*
}

En este artículo se analiza la actual coyuntura interna y externa en los diversos paises de América Latina y se examina si ella clausura las perspectivas de crecimiento de la region en el presente decenio o si, por el contrario, brinda condiciones y oportunidades para retomar la senda del crecimiento.

Se analiza también la literatura cconónica reciente sobre crecimiento y desarrollo en relación con estos problemas y se concluye que la restricción externa, si bien es un problema importante, no determinará por sí sola las perspectivas de crecimiento de la región en el futuro.

Se señala que debería hacerse hincapié en la me. jorfa de diversas condiciones internas, de modo de aumentat la productividad y el crecimiento de la economía nacional. La competitividad aparece como la fuerza impulsora que facilitará la integración en la economia mundial, y ésta a su vez contribuiráa a aumentarla. Las exportaciones, que deben desempeñar un papel dinámico fundamental, dependen de un conjunto de elementos que impulsan el proceso de crecimiento, más que de politicas sectoriales. La dicotomia tradicional entre sustitución de importaciones y promoción de exportaciones será superada con enfoques que combinen to mejor de ambos aspectos.

Por último, se hace presente la necesaria compatibilidad entre la distribución del ingreso y el crecimiento $y$, en una visión divergente de la que privilegia los factores exógenos en el proceso de desarrollo, se destaca que existe un número importante de factores al alcance de la acción de las autoridades económicas.

* Jefe del Programa de investigación del Centro de desarrollo de la Organización para la Cooperación y el Desarrollo Fconómicos (ox.DE), en París. Este estudio fue publicado también en E. Emmery y F. Iglesias, Restoring Financial Flows to Latin America, P'aris, Centro de desarrollo, ocile, 1991.

\section{Introducción}

Que los años ochenta fueron una "década perdida" para América Latina es hoy opinión generalizada. La herencia de esa década es la deuda externa acumulada, que se cierne como una carga y una posible limitación sobre el horizonte de América Latina en el siguiente decenio. Además, es probable que la disponibilidad de nuevos recursos financieros externos se vea fuertemente restringida en los años noventa. La rivalidad por obtener recursos externos será mayor; ahora los Estados Unidos, Europa oriental, la Unión Soviética y los países de Asia sudoriental (ASEAN) competirán por estos fondos, en tanto que en el decenio de 1970 América Latina se mantuvo prácticamente solitaria en su condición de prestatario bien dispuesto y fiable. Las cuestiones sociales, la pobreza y la equidad adquieren un mayor grado de prioridad en las políticas económicas de América Latina que en años anteriores.

Estas diversas presiones sobre América Latina llevan a una pregunta fundamental: si la región podrá recuperar el crecimiento económico en el decenio de 1990.

Lo que se pretende en el presente artículo es determinar hasta qué punto la coyuntura actual, con sus presiones externas e internas, crea circunstancias que cierran el paso a las perspectivas de crecimiento de América Latina en el decenio de 1990, como creen muchos observadores, y en qué medida hay condiciones y oportunidades que gran parte de América Latina pueda capitalizar a fin de iniciar una recuperación del crecimiento en ese decenio. En particular, el autor se basa en algunas publicaciones recientes que tratan del crecimiento y el desarrollo económicos en situación de presiones internas y externas, para evaluar a su vez las posibilidades de recuperar el ritmo de crecimiento en la región. 


\section{Exportaciones impulsadas por el crecimiento o crecimiento impulsado por las exportaciones}

Ultimamente se ha vuelto a examinar en el campo de la economía lo que entendemos por proceso de crecimiento. Varios de los temas abordados en estas publicaciones relativamente nuevas se vinculan entre sí y, lo que es más importante para nuestros propósitos en este caso, afectan nuestra manera de pensar acerca de las políticas económicas que inciden en el crecimiento. Uno de los temas en cuestión es la importancia de la acumulación cuantitativa de los insumos de los factores frente a su mejoramiento cualitativo, y la importancia del capital humano frente al capital físico. Otro tema es la importancia del cambio tecnológico, la manera de tratarlo desde el punto de vista analítico, y la determinación exógena o endógena de la tecnología. Un tercer tema es el de saber si el crecimiento de las exportaciones está determinado por la oferta o si lo impulsa la demanda: en otras palabras, se trata de establecer si los casos de crecimiento dinámico son ejemplos de exportaciones impulsadas por el crecimiento $o$ de crecimiento impulsado por las exportaciones.

Las últimas publicaciones sobre estos temas aclaran la perspectiva conceptual básica a la luz de la cual se formulan las estrategias de desarrollo: si se trata de un proceso de reforma estructural de la economía nacional con fines competitivos en el plano interno, que se traduce en un crecimiento dinámico y una mayor oferta para la exportación; o bien si se trata de un proceso de reforma de las políticas comerciales con fines de competitividad internacional, que permite que la economía reaccione ante la demanda externa. Aunque muchas de las reformas específicas en materia de políticas serían las mismas con arreglo a una u otra formulación, las opciones dan lugar a diferencias considerables en cuanto a la manera de concebir y ejecutar el proceso de desarrollo: por ejemplo, entre lograr la integración en la economía mundial a través del comercio, o lograr la integración económica nacional y el dinamismo mediante una mayor competitividad.

Elhanan Helpman (1988, p. 6) planteo muy bien el problema. Dijo que se necesita una teoría que aborde cuestiones fundamentales como la de determinar si el crecimiento impulsa al comercio o si existe una vinculación inversa que va del comercio al crecimiento; que muchos autores han hecho hincapié en el papel del libre comercio para fomentar el crecimiento, aunque también hay argumentos que destacan el papel determinante de las políticas comerciales en el fomento del rápido crecimiento en Japón y algunos de los países de industrialización reciente; $y$, por último, que la teoría actual no es apropiada para tratar de manera satisfactoria estos distintos criterios.

Los modelos convencionales con rendimientos decrecientes del capital llevaron a formular la hipótesis de la convergencia entre los países. La idea era que se produciría una convergencia entre las tasas salariales y la relación capital-trabajo, puesto que la tasa de rentabilidad de la inversión y la tasa de crecimiento del producto por habitante constituyen funciones decrecientes del nivel de capital nacional por habitante. Puesto que hemos sido testigos de un mundo de crecimiento divergente en el decenio de 1980, parece apropiado volver a examinar el asunto.

En un artículo citado con frecuencia, Paul Romer dijo que si era válida la hipótesis en los modelos convencionales de rendimientos constantes a escala en la producción, el crecimiento del producto estaría totalmente explicado por el crecimiento cuantitativo de los insumos de los factores. Hizo ver que en el trabajo de Kendrick (1976), dando cuenta del crecimiento, se determinó que la tasa de crecimiento del producto se situaba entre 1.06 y 1.30 veces la tasa de crecimiento de los insumos del período de 1929 a 1969 , y ello constituía una prueba de que las tasas de crecimiento de los insumos humanos y no humanos, tangibles e intangibles, no bastaban para explicar el crecimiento del producto (Romer, 1986, p. 1013).

Esto sugiere a Romer la posibilidad de una nueva formulación que entraña tres supuestos. En primer lugar, los conocimientos se consideran 
un bien de capital con un producto marginal cada vez mayor. Se supone que el capital social es un bien compuesto, donde el componente de conocimientos tiene rendimientos de escala crecientes que compensan los rendimientos decrecientes del componente físico del acervo de capital. En segundo lugar, el cambio tecnológico se determina de manera endógena como cualquier otro insumo, con un precio y un producto marginal que establecen su función económica, y no de manera exógena como en la mayoría de los modelos. En último lugar, la rentabilidad de los nuevos conocimientos no es captada únicamente por la empresa que la genera, sino que constituye un efecto externo -o externalidad-. que puede ser recogido por otros agentes económicos. Esto crea una divergencia entre el rendimiento social y el privado que es preciso considerar.

Estas hipótesis representan importantes diferencias respecto de cómo se entiende y activa el proceso de crecimiento y se han tomado como base de trabajos futuros que emprenderán otras personas.

Por ejemplo, Jaime de Melo y Sherman Robinson (1990) han hablado de tres tipos de efectos externos de los conocimientos que contribuyen a explicar el dinamismo del proceso de crecimiento asociado con una elevada relación entre las exportaciones y el producto interno bruto y entre las importaciones y el producto interno bruto. Se trata de efectos externos que tienen que ver con el fomento del comercio, las importaciones y las exportaciones. Los efectos externos del fomento del comercio derivan de las acciones de los gobiernos a través de servicios de información, asesoramiento en materia de creación de mercados, asistencia en materia de diseño y embalaje, etc. Las empresas con ambiciones de exportar pueden aprovecharlos. Las externalidades derivadas de las importaciones son el resultado de las oportunidades de aprender que brinda la importación de bienes de capital y bienes intermedios con tecnologías incorporadas. Las externalidades derivadas de las exportaciones son aquellas que se acumulan al cumplir con las normas de los mercados internacionales y con especificaciones y criterios de calidad, y al solucionar problemas de distribución y comercialización; una vez logradas, se pueden hacer extensivas a otros productos y procesos.
Según De Melo y Robinson, cuando su modelo incluía mediciones de estas externalidades en vez de depender exclusivamente de la acumulación de los factores y del cambio tecnológico exógeno, la relación entre las exportaciones y el PlB y entre las importaciones y el PIB, se ajustaba más a las modalidades de cambio estructural en la producción y el comercio en que exhiben la República de Corea y otro país de industrialización reciente.

Estos resultados y las teorías en que se basaban llevaron a concebir dé manera diferente los beneficios de una orientación hacia el exterior y una apertura hacia la economía mundial. Las ventajas en este caso son la generación de conocimientos y los efectos indirectos producidos por la experiencia ganada en materia de exportaciones, por la importación de tecnologías incorporadas y por los eslabonamientos de la promoción del comercio. Los conocimientos que genera el comercio complementan el acervo de conocimientos adquiridos de fuentes internas: educación, crecimiento económico anterior, desarrollo científico $y$ tecnológico, fomento de la investigación y de su aplicación etc. Si los conocimientos son decisivos para el crecimiento, como sostiene Romer, el hecho de que gran parte del acervo mundial de conocimientos se halle en los paises industriales crea en otros países una necesidad imperiosa de abrirse al comercio, como medio de captar conocimientos del exterior y asimilarlos. En otro trabajo Romer (1989), utilizando una regresión de 90 países, llega a la conclusión de que la apertura, medida por la proporción exportaciones/PIB, eleva la tasa de cambio tecnológico y la productividad marginal del capital, mientras que los incrementos exógenos del ahorro y las inversiones tienen efectos menos poderosos sobre estas variables.

La innovación conceptual en este caso hace algo más que colocar el término " $\mathrm{A}$ " del residuo de la función de producción dentro del paréntesis de la propia función de producción. Hace explícitos el carácter endógeno del proceso de generación de conocimientos, los efectos dinámicos que habrán de obtenerse de este proceso a causa del aumento de la rentabilidad y la calidad de "bien público" de los conocimientos, lo que significa que las empresas y las instituciones tendrán que elaborar estrategias para captar las externalidades. La naturaleza endógena del proce- 
so de absorción de conocimientos mediante la apertura genera un crecimiento económico nacional dinámico que luego produce un crecimiento rápido de la oferta de productos de exportación. Esto no es lo mismo que un crecimiento inducido por las exportaciones e impulsado por la demanda, que resulta primordialmente de hacer que las condiciones del mercado interno reflejen los precios internacionales. En la nueva formulación, la asimilación de conocimientos estimula el crecimiento dinámico de la economía interna, y proporciona de esta manera una oferta excedentaria de bienes exportables. Estas teorías más recientes del crecimiento encajan bastante bien con las modalidades de exportación inducidas por las inversiones, impulsadas por el cambio estructural y fomentadas por la oferta, que de- rivan de la investigación empírica sobre los países de industrialización reciente en diferentes regiones durante el decenio de 1980 (Bradford, 1987).

Helpman (1988), por su parte, concluye que el crecimiento sumamente rápido de las exportaciones de algunos países de industrialización reciente hace pensar que el comercio internacional desempeña un papel importante en el proceso; que puede sostenerse, sin embargo, que el movimiento paralelo del producto y las exportaciones se origina en fuentes internas que producen el crecimiento del producto, el que a su vez induce el crecimiento de las exportaciones, y que la relación de causalidad va del crecimiento hacia el comercio, más bien que en el otro sentido (pp. 15 y 16).

II

\section{Nuevo análisis de la industrialización, el tamaño del mercado y el "gran impulso"}

Muy aparte de las preocupaciones por explicar el rápido crecimiento del comercio, algunos artículos en publicaciones recientes se han remontado a temas e ideas de un período anterior, que han estado al margen de la corriente principal del pensamiento sobre el desarrollo durante 30 años, y los han examinado de nuevo a la luz de reflexiones más recientes. Esos artículos analizan temas como los eslabonamientos intersectoriales, el tamaño de los mercados y los rendimientos crecientes de inversiones simultáneas, los que se trataron inicialmente en publicaciones sobre el crecimiento equilibrado y la industrialización basada en un gran impulso inicial, que datan de epocas tan remotas como los decenios de $1940 \mathrm{y}$ 1950.

Estos temas se examinan en dos artículos de Kevin Murphy, Andrei Shleifer y Robert Vishny (1989a y 1989b). El primero incorpora la idea del rendimiento creciente como elemento principal. De hecho, define la industrialización como la sustitución de las tecnologías de rendimientos constantes por tecnologías de rendimientos crecientes en la producción de algunos bienes (1989a, p. 542). La situación intersectorial que relata es de cierto modo bastante conocida; en ella el crecimiento de la agricultura contribuye de manera importante a generar una demanda suficiente para hacer viabile la industria manufacturera nacional desde una perspectiva económica.

El complemento de esta idea, desde el punto de vista de la oferta, lo suministra la interesante conclusión a que llegaron Jean-Glaude Berthélemy y Christian Morrisson (1989, p. 9): la oferta de bienes manufacturados constituye para los agricultores un factor critico de motivación para generar bienes agrícolas. Si no hay bienes manufacturados que comprar, los agricultores carecen de incentivos para producir más porque no tienen en qué gastar sus ingresos. De esto se deduce que una escasez de bienes manufacturados (bienes de consumo e insumos) puede anular rápidamente los esfuerzos de desarrollo agrícola y llevar a un proceso acumulativo en que las crisis en los sectores agrícola y no agrícola se incentiven mutuamente y en que el sector no agrícola resulte gradualmente paralizado por la caída de las importaciones que traería consigo la disminución de las exportaciones agrícolas. 
Sin embargo, desde el punto de vista de la demanda, Murphy, Shleifer y Vishny (1989a) sostienen que la distribución del ingreso no sólo es compatible con la industrialización, sino que le es necesaria, y citan al respecto cifras de Chenery, Robinson y Syrquin que indican que de $80 \%$ a $90 \%$ de la producción manufacturera de los países en desarrollo se destina al mercado interno. Las industrias que utilizan tecnologias de rendimiento creciente, que a menudo requieren elevados costos fijos en materia de investigación y desarrollo, o de bienes de capital, o ambas cosas a la vez, ven con frecuencia dificultado su avance por falta de una demanda suficiente.

La vieja argumentación de que una mejora en la distribución del ingreso erosionaria las fuentes internas de ahorro - al reducir la participación en el ingreso de los grupos de altos ingresos con mayor propensión al ahorro y elevar la de los grupos de menores ingresos con menor propensión a ahorrar- ha sido rebatida con pruebas y conductas recientes. Una mejor percepción de la importancia del ahorro encubierto en el sector informal, que abarca más del $30 \%$ del empleo no agricola total de América Latina (Turnahm y otros, 1990), y las muestras de que ha habido fuga de capitales y un comportamiento "rentista" entre los latinoamericanos acaudalados, socavan el argumento del ahorro como justificación del crecimiento con desigualdades (Armendáriz de Aghion, 1990).

Murphy, Shleifer y Vishny (1989a, p. 560) dicen que tanto para una economía cerrada como para una economía con algunos mercados abiertos, su análisis destaca el papel que cumple en la industrialización la composición de la demanda determinada por la distribución del ingreso. Sehalan que ese análisis confirma el papel de los sectores principales - como el agrícola o el de exportación-, en el impulso a la industrialización, pero que a la vez muestra que el auge de un sector importante puede no ser suficiente. Para que la industrialización tenga lugar, afirman, los beneficios de tal auge deben distribuirse de manera bastante equitativa como para crear grandes mercados para las manufacturas internas; centrar la atención en la rentabilidad creciente y el tamaño de los mercados internos parece ofrecer elementos de juicio que serían difíciles de obtener de otra manera.
En un segundo artículo (1989b), que de nuevo se centra menos en el comercio que en las causas del atascamiento de la industrialización, los mismos tres autores analizan cómo se puede superar la existencia de un mercado interno pequeño que desalienta las inversiones de empresas cuyas utilidades, si invirtieran por sí solas, no justificarían la inversión global exigida por tecnologías de rendimiento creciente. El problema, entonces, es de externalidades pecuniarias, en contraposición con las externalidades tecnológicas en que hace hincapié Romer.

La solución es simple y bastante conocida. Si varias empresas invierten simultáneamente, los ingresos aumentan lo suficiente como para dar al mercado una dimensión que permita a cada empresa obtener utilidades que justifiquen su inversión; esto no habría sido posible con inversiones aisladas. El argumento se extiende a la infraestructura, donde el bien público se amortiza durante el periodo en que fluye una corriente de ingresos de inversiones múltiples simultáneas, haciéndola factible. El criterio acumulativo, en cambio, llevaría a un equilibrio a nivel más bajo.

Este tipo de razonamiento lleva a la conclusión de que las inversiones simultáneas en múltiples sectores llevan a una trayectoria de crecimiento rentable, económicamente viable y más dinámica, en que pueden darse rendimientos crecientes, y que del mismo modo se pueden ejecutar proyectos de infraestructura compartidos que así se hacen económicamente viables, en tanto que resultarian imposibles si las inversiones se evaluaran y ejecutaran por separado. Las exter. nalidades pecuniarias que convierten este análisis en realidad pueden incorporarse en virtud de un criterio intersectorial, que busque la manera de captar estas externalidades y los beneficios resultantes de las tecnologías de rendimiento creciente. Para estos autores, el factor decisivo es la importancia abrumadora de la demanda interna para la mayor parte de la industria nacional (Murphy y otros, p. 1007). Resulta interesante observar que en un estudio del Instituto Nacional de Estadística, Geografía e Informática de México, entre las empresas que no estaban dispuestas a invertir en ese país en 1988, el $35 \%$ de los encuestados mencionó la incertidumbre en cuanto al tipo de cambio y el $45 \%$ una demanda futura insuficiente. 


\section{III}

\section{Conclusiones: consecuencias para América Latina en el decenio de 1990}

Los artículos citados aportan perspectivas interesantes al estudio de las posibilidades de recuperar el ritmo de crecimiento económico en América Latina en los años noventa. La conclusión general más interesante que quizá pueda extraerse es que si los elementos teóricos medulares en estos artículos se constituyesen de hecho en poderosas fuerzas impulsoras del crecimiento futuro, las limitaciones financieras externas al parecer no determinarían inexorablemente las posibilidades de crecimiento de la región en el próximo decenio. En otras palabras, el hecho de que el panorama sea sombrío en cuanto a atraer corrientes financieras significativas hacia América Latina en los años noventa no anula necesariamente las posibilidades de crecimiento de la región, aunque sí tiene importancia.

En esos aportes se hace hincapié en lograr la trayectoria interna correcta como medio de mejorar la productividad y el crecimiento de la economía nacional y se ponen de relieve los mecanismos y agentes internos que pueden influir en la economía nacional. La competitividad se concibe como el elemento central de un proyecto económico nacional que genera una economía interna más dinámica. Esta competitividad no puede lograrse sin referencia a los precios internacionales ni sin competir con ellos mediante la apertura de la economía, como tampoco sin acceso a tecnologías externas a través de las actividades de importación y exportación. La competitividad económica a nivel nacional facilita la integración en la economía mundial, y viceversa. Pero la razón de ser de la estrategia de desarrollo y su fuerza impulsora se hallan en la competitividad y dinamismo de la economía nacional, que crea sus propios imperativos de apertura, competencia, desregulación, privatización, estabilidad macroeconómica y reforma estructural, y no en las exportaciones como la condición sine qua non del desarrollo.

La diferencia de énfasis es fundamental. Centrar la atención en las exportaciones y las reformas comerciales que ellas necesitan consti- tuye solamente un aspecto de la situación. La idea de un crecimiento inducido por las exportaciones se fundamenta en el concepto de que, si se dan las condiciones apropiadas, habrá exportaciones, pero la teoria no especifica cuáles son los agentes para lograr un crecimiento dinámico de las exportaciones, más allá de señalar los beneficios que en materia de eficiencia se obtienen de los efectos distributivos estáticos de obtener precios justos. La idea de que las exportaciones sean inducidas por el crecimiento se sustenta en una gama más rica de elementos que activan el proceso de crecimiento. Estos se centran en el proceso de generación de conocimientos, tanto interno (merced a la educación, la capacitación, la alfabetización, el apoyo a la investigación y desarrollo, etc.) como respecto de la fundamental absorción de tecnologías del exterior por conducto de políticas de apertura económica. El aprendizaje por la práctica, la inversión en tecnologías de rendimientocreciente en la industria, la consecución de tecnologías incorporadas a través de las importaciones, la adquisición de experiencia en materia de exportaciones y el aprovechamiento de los efectos externos de la promoción comercial, proporcionan en conjunto, en esta conceptualización del proceso, elementos de dinamismo económico.

Frente al concepto de que las exportaciones son inducidas por el crecimiento, los agentes económicos tienen más que hacer. La insistencia en que la economía nacional se ponga en marcha por razones internas vivifica y arraiga el proceso de reformas en el marco económico, institucional y político del país, donde tiene más prominencia y fuerza. La apertura de la economía a la competencia internacional es parte de un compromiso de fortalecer las reformas internas a fin de aumentar la competitividad nacional y es, a su vez, impulsada por este compromiso. La formulación de políticas de crecimiento inducido por las exportaciones aún es vital, pero ahora se inserta en un marco más amplio y complejo que incumbe a toda la sociedad. Durante mucho tiem- 
po nos hemos visto inmersos en debates acerca de opciones dicotómicas estrechas entre sustitución de importaciones y vocación exportadora, en vez de abocarnos a la búsqueda de una combinación de elementos (Calvino, 1988, p. 20). Un buen ejemplo de este enfoque "combinatorio" se encuentra en el reciente estudio titulado Transformación productiva con equidad (CEPAL., 1990, en especial las pp. 85 y 102 a 104).

EI segundo elemento que aflora del presente examen de publicaciones recientes es la confianza renovada en la posible compatibilidad entre la distribución del ingreso y el crecimiento, que da más importancia al tamaño del mercado interno y a los enfoques intersectoriales para estimular el crecimiento. Estos factores aportan otro conjunto de elementos positivos para que las economías grandes y medianas de América Latina enfrenten los dilemas y desafíos en materia de política en los años noventa.

En conjunto, esta diversidad de argumentos permite vislumbrar un panorama positivo para
América Latina en el futuro. Los diversos elementos tienen como característica común el hallarse plenamente al alcance de la región. Los agentes económicos nacionales pueden actuar para hacer realidad las potencialidades de la economía nacional mediante la asimilación del conocimiento y la innovación tecnológica del exterior, y para aprovechar las ventajas de un gran mercado interno. Naturalmente, el ámbito financiero y comercial externo sigue teniendo importancia, pero no es necesariamente determinante. Si la expansión dinámica de las exportaciones es determinada en gran medida por la oferta y es más el resultado que la causa de un rápido crecimiento económico, la tarea y las posibilidades de recuperar el crecimiento de la región recaen más bien en la propia América Latina, y están libradas a fuerzas económicas de origen exógeno. Esto puede considerarse positivo para una región que se aboca cada vez más a la tarea de poner en orden sus asuntos internos por razones propias, en momentos en que la democracia retorna a ella.

\section{Bibliografia}

Armendáriz de Aghion, Beatriz (1990): Income distribution and domestic savings mobilization in Latin America, París, Centro de desarrollo de la Organización para la Cooperación y el Desarrollo Económicos (OCDE), mimeo.

Berthélemy, J.C. y C. Morrisson (1989): Agricultural Development in Africa and the Supply of Manufactured Goods, Paris, Centro de Desarrolto, Organizacion para la Cooperación y el Desarrollo Económicos (or:ue).

Bradford, Colin I., Jr. (1987): Trade and strucuural change: NICs and next tier NICs as ttansitional economies, World Deolopment, vol. 15, N" 3, Nueva York, Pergamon Journals Inc.

Galvino, Italo (1988): Six Memos for the Next Millennium, Cambridge, Mass, Harvard University Press.

CEML.(Comisión Económica para América Latina y el Caribe) (1990): Transformación productiva con equidad. La tarea prioritaria del desamollo de América Latina y el Caribe en las años nowenta. Santiago de Chile. Publicación de las Naciones Unidas, N" de venta: s.90. II.c.6.

Davrieux, Ariel (1990): Prospects for the re-establishment of economic growth in South America, documento preparado para el International Forum on Latin American Perspectives, organizado conjuntamente por el Banco Interamericano de Desarrollo (ви) y el Centro de desarrollo de la Organización para la Cooperación y el Desarrollo Econónicos (o(D).), París, 21-23 noviembre de 1990.
De Melo, Jaime y Sherman Robinson (1990): Productivity and Externalities: Models of Export-Led Growth, PPR Working Papers, wPs 387, Washington, D.C., Banco Mundial.

Dervis, Kemal y Peter A. Petri (1987): The macroeconomics of successful development: what are the lessons?, NBER Macroeconomics Annual 1987, Cambridge, Mass., MIT Press.

Grossman, Gene M. y Elhanan Helpman (1989a): Comparative advantage and long-run growth, NBER Working Paper N" 2809, Cambridge, Mass., National Bureau of Economic Research.

(1989b): Endogenous product cycles, NBER Working Paper N" 2913, Cambridge, Mass., National Bureau of Economic Research.

$(1989 \mathrm{c})$ : Growth and welfare in a small open economy, NBE R Working Paper N" 2970 (revisado), Cambridge, Mass., National Bureau of Economic Research.

(1989d), Quality ladders and product cycles, NBER Working Paper N" 3201, Cambridge, Mass., National Bureau of Economic Research.

Helpman, Elhanan (1988): Growth, technological progress and trade, NBER Reprint No 1145, Cambridge, Mass., National Bureau of Economic Research.

Murphy, Kevin M., André Shleifer and Robert Vishny (1989a): Industrialization and the big push, Joumal of Political Economy, vol. 97, N" 5, Chicago, Illinois, The University of Chicago Press. 
(1989b): Incone, distribution, market size, and industrialization, Quarterly Journal of Economics, wol. c15, N"3, Cambridge, Mass, , MT Press, agosto.

Romer, Paul M. (1986): Increasing returns and bong-run growth, Joumal of Polatical Economy, vol. 94, N" 5, Chicago, Illinois, The University of Chicago Press, octubre.

(1987): Crazy explanations for the productivity slowdown, NBER Macroeconomics Amnual 1987, Cambridge, Mass, MrT Press.

(1989): What Determines the Rate of (irowth and
Technological Change?, l'PR Working Papers, w'rs 279 , Washington, D.C., Banco Mundial.

'Tumham, D., B. Salomé y A. Schwarz (comps.) (1990): The Informal Sector Revisited, Pat'is, Centro de Desarrollo, Organización para la Cooperación y el Desarrollo Económicos (OC:DE).

Williamson, John (comp.) (1990): Latin American Adjustment: How Much Has Happened?, Cambridge, Mass. y Washington, D.C., wrr Press, Institute for International Economics. 